\title{
Equation of State Constraints from the Threshold Binary Mass for Prompt Collapse of Neutron Star Mergers
}

\author{
Andreas Bauswein $\odot,{ }^{1}$ Sebastian Blacker $\odot,{ }^{1,2}$ Vimal Vijayan, ${ }^{1}$ Nikolaos Stergioulas $\odot,{ }^{3}$ Katerina Chatziioannou, ${ }^{4}$ \\ James A. Clark, ${ }^{5}$ Niels-Uwe F. Bastian $\odot,{ }^{6}$ David B. Blaschke $\odot,{ }^{6,7,8}$ Mateusz Cierniak, ${ }^{6}$ and Tobias Fischer $\odot^{6}$ \\ ${ }^{1}$ GSI Helmholtzzentrum für Schwerionenforschung, Planckstraße 1, 64291 Darmstadt, Germany \\ ${ }^{2}$ Institut für Kernphysik, Technische Universität Darmstadt, 64289 Darmstadt, Germany \\ ${ }^{3}$ Department of Physics, Aristotle University of Thessaloniki, 54124 Thessaloniki, Greece \\ ${ }^{4}$ Center for Computational Astrophysics, Flatiron Institute, 162 th Ave, New York, New York 10010, USA \\ ${ }^{5}$ Center for Relativistic Astrophysics, School of Physics, Georgia Institute of Technology, Atlanta, Georgia 30332, USA \\ ${ }^{6}$ Institute of Theoretical Physics, University of Wrocław, 50-205 Wroctaw, Poland \\ ${ }^{7}$ National Research Nuclear University (MEPhI), 115409 Moscow, Russia \\ ${ }^{8}$ Bogoliubov Laboratory for Theoretical Physics, Joint Institute for Nuclear Research, 141980 Dubna, Russia
}

(Received 1 April 2020; revised 15 May 2020; accepted 25 August 2020; published 30 September 2020)

\begin{abstract}
Using hydrodynamical simulations for a large set of high-density matter equations of state (EOSs), we systematically determine the threshold mass $M_{\text {thres }}$ for prompt black-hole formation in equal-mass and asymmetric neutron star (NS) mergers. We devise the so far most direct, general, and accurate method to determine the unknown maximum mass of nonrotating NSs from merger observations revealing $M_{\text {thres }}$. Considering hybrid EOSs with hadron-quark phase transition, we identify a new, observable signature of quark matter in NS mergers. Furthermore, our findings have direct applications in gravitational wave searches, kilonova interpretations, and multimessenger constraints on NS properties.
\end{abstract}

DOI: 10.1103/PhysRevLett.125.141103

Motivation and context.-With the sensitivity increase of current gravitational-wave (GW) detectors, observations of neutron star (NS) mergers will become routine in the very near future $[1,2]$. Also, the identification of electromagnetic counterparts will succeed frequently as sky localizations from the GW signal improve, more dedicated instruments become operational and observing strategies advance. This includes the radiation from ejecta in the ultraviolet, optical and infrared wavebands, so-called kilonovae [3], but also gamma ray, x-ray, and radio emission from relativistic outflows [4].

One of the most basic features of an NS coalescence is the immediate merger product, which can be either a black hole $(\mathrm{BH})$ for high total binary masses or an NS remnant for lower total masses [5-8]. The latter may undergo a delayed collapse to a BH. Generally, the NS remnant's lifetime increases with decreasing total binary mass [9-17].

Based on the distinction between prompt and delayed BH formation for systems with different total binary mass, one can introduce a threshold binary mass $M_{\text {thres }}$ for direct collapse, which is measurable. The total binary mass $M_{\text {tot }}$ can be inferred with good precision from the inspiral GW signal, i.e., the premerger phase (in practice, the chirp mass is measured with very high precision and constraints on the binary mass ratio are required to obtain $\left.M_{\text {tot }}[1,18]\right)$. The merger outcome can be observationally discerned either by the presence of strong postmerger GW emission from an NS remnant $[19,20]$ (absent for direct $\mathrm{BH}$ formation) or from the properties of the electromagnetic counterpart, which is expected to be relatively dim for prompt-collapse events because of reduced mass ejection [21-23]. Thus, a number of measurements with different $M_{\text {tot }}$ and information on the merger product yields $M_{\text {thres }}$. The measurement uncertainty essentially depends on how the detections sample the $M_{\text {tot }}$ range. It should thus continuously decrease with the number of events which allow a distinction between the possible outcomes.

The threshold binary mass is highly important for the interpretation of NS merger observations [2,3,18,23-36]. Moreover, $M_{\text {thres }}$ depends in a specific way on the incompletely known equation of state (EOS) of NS matter [8]. Therefore, understanding the EOS dependence of the collapse behavior is crucial for current and future constraints on unknown properties of high-density matter and of NSs, such as their maximum mass [8], radii [37-39], and tidal deformabilities $[13,40-42]$. The prospect to determine $M_{\max }$ is very notable, where solid lower limits are currently provided by pulsar measurements [43-45]. Upper limits are inferred through more elaborated interpretations of observational data indicating a finite remnant lifetime, e.g., [23,46-55]. Estimates of the remnant's late-time behavior may be subject to considerable model dependencies. The present Letter instead deals with the threshold for direct $\mathrm{BH}$ formation, which leads to different, relatively strong and clear observational features. Apart from implications for high-density matter physics [56-58], $M_{\max }$ is also 
crucial for $\mathrm{BH}$ formation in core-collapse supernovae and BH physics [59-64].

In this Letter, we determine the impact of the properties of high-density matter on the threshold binary mass for prompt $\mathrm{BH}$ collapse. We go beyond current knowledge in several respects. We consider the so far largest sample of EOSs to determine $M_{\text {thres }}$ and find new, tight relations describing its EOS dependence. For the first time, we systematically determine binary mass ratio effects on $M_{\text {thres }}$. Furthermore, we investigate the impact of phase transitions on the collapse behavior.

We put forward the following four main applications of our new findings:

(1) Along the lines of [8], we devise a more direct, more general, and more accurate method to determine $M_{\max }$ from NS merger observations providing information about the immediate merger outcome and about the total binary mass and combined tidal deformability from the inspiral GW signal.

(2) We identify a new signature of a phase transition to deconfined quark matter in NS mergers. This stresses the enormous potential of future merger observations [65-86] to understand the phase diagram of matter in the nonperturbative regime of finite chemical potentials, which is not accessible by $a b$ initio QCD calculations $[87,88]$. Currently, it is not known whether the hadron-quark phase transition takes place at typical NS densities. Identifying an imprint of the phase transition in merger observables will thus also provide invaluable insights for heavy-ion experiments, which will explore the phase diagram at such densities and finite temperature (but different isospin) [89-91].

(3) Beyond these future prospects, our novel, more general relations are directly applicable in detection and analysis pipelines to quantify the likelihood of a specific merger outcome and thus, for instance, GW and kilonova characteristics $[2,3,23,26,30-34,92-96]$.

(4) Furthermore, they are key input for current multimessenger constraints on NS properties as in [37-42].

See, e.g., [23,26,27,30-33,37-39,94-96] for concrete implementations of $M_{\text {thres }}$ dependencies. These applications can be significantly improved by the findings in this study. Below we describe our results mostly in the context of the first two applications.

Simulations and setup.-We perform three-dimensional relativistic hydrodynamical simulations of NS mergers for a large set of different EOSs of NS matter. For every EOS, we compute $M_{\text {thres }}(\mathrm{EOS} ; q)$ for fixed binary mass ratios $q=M_{1} / M_{2}=1$ and $q=0.7$. Masses $M_{1}$ and $M_{2}$ of the individual binary components, $M_{\text {tot }}$ and $M_{\text {thres }}$ refer to the gravitational mass (for binaries at infinite orbital separation). Simulations start from quasiequilibrium circular orbits a few revolutions before merger, with stars initially at zero temperature and in neutrino-less beta equilibrium. The merger calculations are conducted with a relativistic smooth particle hydrodynamics code, which adopts the spatial conformal flatness condition to solve the Einstein field equations [97,98]. More details on the simulation tool, comparisons to other codes (showing generally a very good agreement), and resolution studies can be found in [8,32,37,38,99-104].

In this study, we consider 23 different hadronic EOSs [62,105-124], which constitute our "base sample" and are consistent with astrophysical constraints from $[1,43,44]$. To enlarge the parameter space, we optionally supplement those with eight additional hadronic EOSs [115-118,125-128] which are incompatible with the tidal deformability constraints from GW170817 [1]. Among all these EOSs, five models include hyperons. Additionally, we consider nine hybrid models with a first-order phase transition to deconfined quark matter leading to a strong softening of the EOS $[68,111,129-132]$. These models vary in the onset density, the latent heat, and the stiffness of quark matter $[68,86]$. Among all 40 EOSs, 26 are fully temperature dependent. The remaining models are supplemented with an approximate treatment of thermal effects [101]. We refer to the Supplemental Material [133], which provides more details on the simulations and the different sets of EOS models and includes Refs. [134-138]. We emphasize that our base sample covers well the full range of viable hadronic models.

$M_{\max }$ determination.-We first consider results for the base sample of purely hadronic EOSs assuming that indications of a strong phase transition to quark matter may be independently provided by other observations or experiments $[68,75,76,89,132]$. We further justify this assumption below by describing a new detectable signature of a phase transition.

Compiling the data for equal-mass mergers, Fig. 1 reveals a tight relation between the maximum mass $M_{\max }$ of nonrotating NSs, the threshold binary mass $M_{\text {thres }}$ and $\tilde{\Lambda}_{\text {thres. }}$. The latter is the combined tidal deformability of

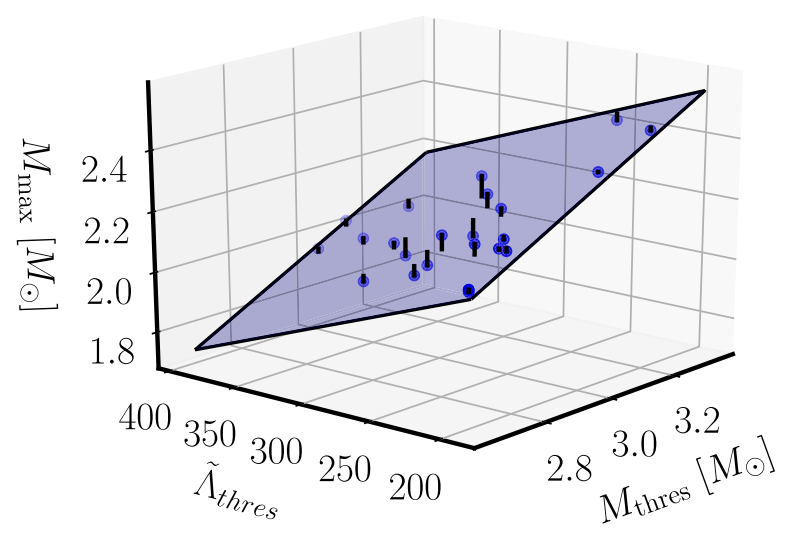

FIG. 1. Maximum mass $M_{\max }$ of nonrotating NSs as function of the threshold binary mass $M_{\text {thres }}$ for prompt BH formation and combined tidal deformability $\tilde{\Lambda}_{\text {thres }}=\tilde{\Lambda}\left(M_{\text {thres }} / 2\right)$ of the binary at the threshold for direct collapse. Blue plane shows a bilinear fit to the data for $q=1$. Short black lines visualize the deviation between fit (blue plane) and data points. 
the binary system at the threshold mass, i.e., $\tilde{\Lambda}_{\text {thres }} \equiv$ $\Lambda\left(M_{\text {thres }} / 2\right)$ for $q=1$. $\tilde{\Lambda}$ describes the dominant EOS effects during the GW inspiral and is thus measurable $[1,18,139-147]$. It is defined by $\tilde{\Lambda}=\left\{16 /\left[13\left(M_{1}+\right.\right.\right.$ $\left.\left.\left.M_{2}\right)^{5}\right]\right\}\left[\left(M_{1}+12 M_{2}\right) M_{1}^{4} \Lambda_{1}+\left(M_{2}+12 M_{1}\right) M_{2}^{4} \Lambda_{2}\right] \quad$ with tidal deformabilities $\Lambda_{1(2)}$ of the individual binary components [148-150]. $\Lambda(M)$ is a stellar structure parameter and fully determined by the EOS through $\Lambda_{1(2)}=$ $\frac{2}{3} k_{2}\left(R_{1(2)} / M_{1(2)}\right)^{5}$ with the tidal Love number $k_{2}(M)$ and stellar radius $R(M)$ (factors of $G$ and $c$ suppressed).

The tidal deformability monotonically decreases with mass. Therefore, $\tilde{\Lambda}_{\text {thres }}$ can be obtained from measurements of systems with different $M_{\text {tot }}$ around $M_{\text {thres }}$ through a simple interpolation. The tight relation in Fig. 1 implies that a sufficiently accurate measurement of $M_{\text {thres }}$ and $\tilde{\Lambda}_{\text {thres }}$ determines the currently unknown maximum mass of nonrotating NSs. The data in Fig. 1 is well described by a bilinear fit

$$
M_{\text {max }}\left(M_{\text {thres }}, \tilde{\Lambda}_{\text {thres }}\right)=a M_{\text {thres }}+b \tilde{\Lambda}_{\text {thres }}+c,
$$

with $a=0.632, b=-0.002 M_{\odot}$, and $c=0.802 M_{\odot}$. The maximum residual of this fit is only $0.067 M_{\odot}$, implying a potentially very accurate measurement of $M_{\max }$ (see the Supplemental Material [133] for fits with an enlarged set of EOSs). The average deviation between Eq. (1) and the underlying data is only $0.02 M_{\odot}$. As an example, assuming $M_{\text {thres }}$ to be measured within $0.05 M_{\odot}$ and $\tilde{\Lambda}_{\text {thres }}$ within $5 \%$, an error propagation through Eq. (1) yields $\Delta M_{\max }=0.06 M_{\odot}$.

For $q=0.7$, we obtain a similarly tight relation for hadronic EOSs with a maximum residual of $0.078 M_{\odot}$ (with fit parameters $a=0.621, b=-0.001 M_{\odot}, c=0.582 M_{\odot}$ ) (see Table II in the Supplemental Material [133]). For the same EOS, $M_{\text {thres }}$ of asymmetric systems is comparable to the one of equal-mass mergers (either equal or at most $0.2 M_{\odot}$ smaller). Moreover, we find the difference in $M_{\text {thres }}$, i.e., $M_{\text {thres }}(q=1)-M_{\text {thres }}(q=0.7)$, to depend systematically on the EOS. See the Supplemental Material [133] for more details, a discussion of the systematic impact of the mass ratio and an intuitive explanation.

Based on our models, we construct additional bilinear fits (Table II in the Supplemental Material [133]) quantifying their quality by the maximum residual and the average deviation between fit and data. For these relations, we select different subsets of our data motivated by different assumptions on which additional information may be available (e.g., about $q$ or the presence of a phase transition). For instance, we consider only purely hadronic EOS models or a full set of EOSs including hybrid models with phase transitions, or we include binaries with a fixed mass ratio or a range in $q$. We also employ different independent variables, which may be measured more precisely in comparison to the quantities in Eq. (1). This includes (i) the chirp mass $\mathcal{M}_{c}=\left(M_{1} M_{2}\right)^{3 / 5} /\left(M_{1}+M_{2}\right)^{1 / 5}$ if the mass ratio is not well constrained or strongly differs among the different events which are combined to determine $M_{\text {thres }}$, (ii) the tidal deformability $\Lambda_{1.4}$ of a $1.4 M_{\odot}$ NS, which may be more accurately and independently measured than $\tilde{\Lambda}_{\text {thres }}$, or (iii) the radius $R_{1.6}$ of a $1.6 M_{\odot}$ NS. We stress that cases (ii) and (iii), i.e., fits 8 to 11 , are very promising when $\Lambda_{1.4}$ or $R_{1.6}$ is measured in a high SNR GW detection or by another astronomical observation, e.g., by NICER [151-153].

Generally, all these choices lead to tight relations describing the collapse behavior. This is not unexpected considering the previously found relation $M_{\text {thres }}=$ $\left[-3.606\left(G M_{\max } / c^{2} R_{1.6}\right)+2.38\right] M_{\max }$ for a smaller set of EOS models and only equal-mass mergers $[8,154]$. NS radii are roughly constant in a considerable mass range around $M_{\text {thres }} / 2$ and the tidal deformability is known to scale approximately with NS radii (see also [154] for a semianalytic model of the collapse behavior). In comparison to previous results, the new relations presented here allow a more direct and more general implementation in analysis pipelines or waveform models because they involve quantities which are directly measurable from the GW inspiral (of the same event) and do not rely on additional information, e.g., about $R_{1.6}$. They also include asymmetric binaries. We remark that the functional form of our new fits like Eq. (1) is more physical compared to relations in [8], which features a unphysical decrease of $M_{\text {thres }}$ with $M_{\max }$ in a very small range of the parameter space. Finally, we directly compare the relations $M_{\text {max }}\left(M_{\text {thres }}, \tilde{\Lambda}_{\text {thres }}\right) \quad\left[\right.$ Eq. (1)] and $M_{\max }\left(M_{\text {thres }}, R_{1.6}\right)$ (inverted relation from [8]). The maximum residual is $0.067 M_{\odot}$ for the new relation compared to $0.26 M_{\odot}$ for the latter. Hence, the relations describing the collapse behavior in this work are significantly more accurate while they even include more models and consider asymmetric mergers.

Physically, relations as Eq. (1) are understandable. $M_{\text {thres }}$ is determined by two roughly independent EOS properties, namely, $\tilde{\Lambda}_{\text {thres }}$ characterizing the EOS stiffness at moderate densities and $M_{\max }$ at very high densities, both of which increase $M_{\text {thres }}$. For fixed $M_{\text {thres }}$, this implies that $\tilde{\Lambda}_{\text {thres }}$ has to decrease with $M_{\max }$.

We emphasize that already a single measurement of $M_{\text {tot }}$ and $\tilde{\Lambda}$ can yield a strong constraint on $M_{\max }$. Indications for a prompt collapse in a detection imply $M_{\text {tot }}>M_{\text {thres }}$ and $\tilde{\Lambda}<\tilde{\Lambda}_{\text {thres. }}$. From this follows through Eq. (1) that the actual maximum mass of nonrotating NSs is smaller than $M_{\max }\left(M_{\mathrm{tot}}, \tilde{\Lambda}\right)$ (note the minus sign of the fit parameter $b$ ). If a measurement provides evidence for no direct $\mathrm{BH}$ formation, the maximum mass of NSs has to be larger than $M_{\text {max }}\left(M_{\text {tot }}, \tilde{\Lambda}\right)$ because $M_{\text {tot }}<M_{\text {thres }}$ and $\tilde{\Lambda}>\tilde{\Lambda}_{\text {thres }}$ (for instance, a prompt (delayed) collapse event with $M_{\text {tot }}=$ $3.0 M_{\odot}$ and $150<\tilde{\Lambda}<250$ implies $M_{\max }<2.40 M_{\odot}$ $\left(M_{\max }>2.20\right)$, which may be further tightened by incorporating additional $\tilde{\Lambda}$ data from other events). 
Further applications.-All aforementioned relations are bilinear and thus easy to invert for other applications requiring for instance $M_{\text {thres }}$ or the tidal deformability to be the dependent quantity (applications 3 and 4). We stress that one can exploit our different relations describing the collapse behavior even if some parameters are poorly constrained as, for instance, in [37-39] yielding a lower bound on NS radii of about $11 \mathrm{~km}$.

Our models also show that the range of $\tilde{\Lambda}_{\text {thres }}$ is relatively large: for equal-mass mergers $200 \lesssim \tilde{\Lambda}_{\text {thres }} \lesssim 450$, whereas $200 \lesssim \tilde{\Lambda}_{\text {thres }} \lesssim 650$ for $q=0.7$ [104], which is significantly broader than previously assumed (cf. $[32,155,156])$. Hence, only for $\tilde{\Lambda}<200$, a prompt collapse can be assumed, while depending on $q$ only events with $\tilde{\Lambda} \gtrsim 650$ may safely be classified as no direct collapse. This is, for example, relevant for kilonova observations and $\mathrm{GW}$ searches to determine whether there may be contributions from strong postmerger $\mathrm{GW}$ emission. These ranges imply that independent of $M_{\max }$ the tidal deformability of a $1.37 M_{\odot}$ NS has to be larger than about 200 following the arguments in Refs. [37,40,42,49] favoring a delayed collapse in GW170817. This limit is less than the one reported in [40,41], but our data clearly show that current observations do not exclude EOSs with $\Lambda_{1.37}>200$ in line with $[37,42,157]$.

New signature of phase transition.-By additionally considering the results with hybrid EOSs, we identify a new observable signature of the hadron-quark phase transition, which may occur in NSs. Figure 2 shows $\tilde{\Lambda}_{\text {thres }}$ as function of $M_{\text {thres }}$ for all EOSs with $q=1$. It is striking that all 31 purely hadronic models are located below the dashed line given by

$$
\tilde{\Lambda}_{\text {thres }}^{\text {hybrid }}=488\left(M_{\text {thres }} / M_{\odot}\right)-1050 \text {, }
$$

whereas most hybrid models with a phase transition occur above this curve, i.e., at relatively small $M_{\text {thres }}$ but larger $\tilde{\Lambda}_{\text {thres }}$. Hence, a combined measurement of $\left(M_{\text {thres }}, \tilde{\Lambda}_{\text {thres }}\right)$ with $\tilde{\Lambda}_{\text {thres }}>488\left(M_{\text {thres }} / M_{\odot}\right)-1050$ provides strong evidence for the presence of a phase transition.

A strong phase transition induces a softening of the EOS at higher densities and thus destabilizes the merger product, i.e., yields a relatively small $M_{\text {thres }}$. For most of these models, $M_{\text {thres }} / 2$ is smaller than the smallest mass $M_{\text {onset }}$ at which quark matter appears in nonrotating NSs. Hence, the inspiralling stars are purely hadronic and the corresponding tidal deformability $\tilde{\Lambda}_{\text {thres }}$ does not carry any information about the phase transition and is thus relatively large. To some extent, this effect is comparable to results in [68], where a stronger compactification of the merger remnant by the phase transition leads to a characteristic increase of the postmerger GW frequency.

Figure 2 is a projection of the data point of Fig. 1 onto the $M_{\text {thres }}-\tilde{\Lambda}_{\text {thres }}$ plane. We can thus draw lines of constant $M_{\max }$ using fit (1) for purely hadronic EOSs. This explains

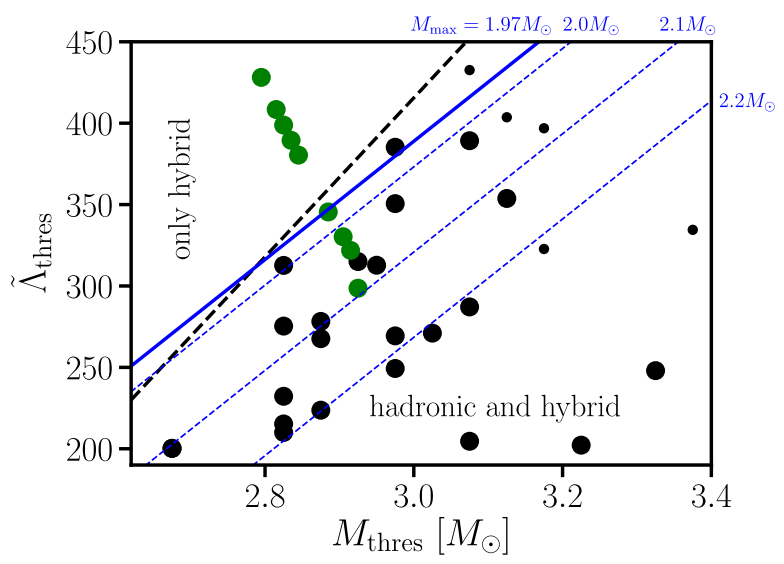

FIG. 2. Combined tidal deformability of binaries at the threshold to prompt $\mathrm{BH}$ formation as function of threshold binary mass $M_{\text {thres }}$ for direct collapse for different hybrid EOSs (green points) and purely hadronic EOSs (black points; small symbols are models of the "excluded" sample, three outside the plot range). Dashed line indicates boundary beyond which only hybrid models exist [Eq. (2)]. In all except for one hybrid model, the phase transition occurs after merger. Hence, $\tilde{\Lambda}_{\text {thres }}=\Lambda\left(M_{\text {thres }} / 2\right)$ is that of purely hadronic stars, which for our models all are described by the same hadronic EOS below the onset density of the phase transition. Therefore, $\tilde{\Lambda}_{\text {thres }}\left(M_{\text {thres }}\right)$ of these hybrid models appear to line up on a single curve, following $\Lambda(M)$ of the hadronic EOS. The blue lines show curves of constant $M_{\max }$ using fit (1) for purely hadronic EOSs explaining the absence of viable hadronic models in the upper left corner.

why no viable purely hadronic EOS models occur above the dashed line: only models with $M_{\max }<1.97 M_{\odot}$ could yield a $\left(M_{\text {thres }}, \tilde{\Lambda}_{\text {thres }}\right)$ combination in the upper left corner, which however is excluded by pulsar mass measurements $[43,45]$. The situation is different for EOSs with a strong phase transition even if they yield a maximum mass above $2 M_{\odot}$. As explained, those models can feature a strong softening at higher densities, which leads to a destabilization of the merger remnant and a correspondingly low $M_{\text {thres }}$. A low $M_{\text {thres }}$ implies that the merging stars of the system with $M_{\text {tot }}=M_{\text {thres }}$ are hadronic and relatively light. This leads to a relatively large $\tilde{\Lambda}_{\text {thres }}$. Thus, hybrid models with this behavior can lead to strong deviations from the $M_{\max }\left(M_{\text {thres }}, \tilde{\Lambda}_{\text {thres }}\right)$ relation of hadronic EOSs and the data points $\left(M_{\text {thres }}, \tilde{\Lambda}_{\text {thres }}\right)$ can occur in a regime inaccessible by viable hadronic models.

This explains our finding and solidifies that the described signature through the criterion in Eq. (2) is indicative of a phase transition. For $q=0.7$, we find a qualitatively similar behavior [104].

Following this argumentation, we further point out that the limit which indicates a phase transition can be updated when pulsar measurements increase the lower bound on $M_{\max }$.

We stress several advantages of this new signature to uncover the hadron-quark phase transition in NS mergers. (1) $\tilde{\Lambda}_{\text {thres }}$ does not need to be determined with very high 
precision in comparison to the accuracy which would be required to detect a relatively weak kink at $M_{\text {onset }}$ in $\Lambda(M)$ indicating a phase transition (e.g., Fig. 3 in [42]). A precision of $10 \%$ to $30 \%$ is sufficient. (2) For most hybrid EOSs studied here, $M_{\text {thres }} / 2<M_{\text {onset }}$, which implies that $\tilde{\Lambda}_{\text {thres }}$ is larger than $\Lambda\left(M_{\text {onset }}\right)$ and thus easier to measure (because of stronger finite-size effects and possibly more frequent systems). Detecting a phase transition with high $M_{\text {onset }}$ becomes increasingly challenging for methods employing only the GW inspiral [75,76], in which case our signature is particularly promising because it is sensitive to the very high-density regime. (3) Already a single measurement with a constraint on $\left(M_{\text {thres }}, \tilde{\Lambda}_{\text {thres }}\right)$ may reveal indications of a phase transition. (4) $M_{\text {tot }}$ can be measured with very good precision and there are a number of different signals potentially revealing the merger product, e.g., postmerger GWs, kilonovae, and possibly gamma-ray bursts, implying that a sufficient $M_{\text {thres }}$ determination is conceivable in the near future. In fact, all in principle required observables have already been measured $[1,4]$.

Notably, not all hybrid EOSs lie in the "hybrid regime" above the dashed line in Fig. 2. These are models with a very strong stiffening of the EOS in the quark phase (with $M_{\max }$ exceeding the one of the purely hadronic reference model; see the Supplemental Material [133]). One may refer to this as a coarse variant of the masquerade problem [121], where hybrid models roughly resemble the massradius relation of purely hadronic EOSs. The stiffening leads to a stabilization of the merger product and thus to a relatively large $M_{\text {thres }}$ and consequently a relatively small $\tilde{\Lambda}_{\text {thres }} \equiv \Lambda\left(M_{\text {thres }} / 2\right)$. A $\left(M_{\text {thres }}, \tilde{\Lambda}_{\text {thres }}\right)$ below the dashed curve does thus generally not allow to infer the nature of high-density NS matter. However, the proximity to the dash curve indicates a softening of the EOS at higher densities and possibly the occurrence of a weak phase transition.

Conclusions. - Future work should investigate potential systematic uncertainties which might exist on a very low level, overcome those by improved numerical and physical modeling, and explore in more detail the observational features resulting from the collapse behavior. Also, an even larger set of hybrid EOSs should be considered because our current models vary the properties of the quark phase but employ the same hadronic EOS at densities below the phase transition. This hadronic reference model lies in the middle of the range given by current astrophysical and experimental constraints $[1,37,43,44,58,147,158-162]$. We thus expect that other hybrid models show the same behavior; such models should essentially be shifted parallel to the dashed line.

We thank G. Martinez-Pinedo and H.-T. Janka for helpful discussions. We thank C. Constantinou, M. Prakash, A. Schneider, and J. Smith for help with their EOS tables. A. B. acknowledges support by the European Research Council under the European Union's Horizon
2020 research and innovation programme under Grant No. 759253. A. B. and S. B. acknowledge support by Deutsche Forschungsgemeinschaft (DFG, German Research Foundation)-Project-ID 279384907-SFB 1245. A. B. and V. V. acknowledge support by DFG-Project-ID 138713538-SFB 881 (“The Milky Way System,” subproject A10). D. B. B. and T. F. acknowledge support from the Polish National Science Center (NCN) under Grant No. 2019/33/B/ST9/03059. T. F. acknowledges support from NCN under Grant No. UMO-2016/23/B/ST2/ 00720. N.-U. F. B. acknowledges support from NCN under Grant No. 2019/32/C/ST2/00556. N. S. is supported by the ARIS facility of GRNET in Athens (SIMGRAV, SIMDIFF, and BNSMERGE allocations) and the "Aristoteles Cluster" at AUTh, as well as by the COST actions CA16214 "PHAROS," CA16104 "GWVerse," CA17137 "G2Net," and CA18108 "QG-MM."

[1] B. P. Abbott, R. Abbott, T. D. Abbott, F. Acernese, K. Ackley, C. Adams, T. Adams, P. Addesso, R. X. Adhikari, V. B. Adya et al. (LIGO Scientific and Virgo Collaborations), Phys. Rev. Lett. 119, 161101 (2017).

[2] B. P. Abbott, R. Abbott, T. D. Abbott, S. Abraham, F. Acernese, K. Ackley, C. Adams, R. X. Adhikari, V. B. Adya, C. Affeldt et al., Astrophys. J. Lett. 892, L3 (2020).

[3] B. D. Metzger, Living Rev. Relativity 23, 1 (2020).

[4] B. P. Abbott, R. Abbott, T. D. Abbott, F. Acernese, K. Ackley, C. Adams, T. Adams, P. Addesso, R. X. Adhikari, V. B. Adya et al., Astrophys. J. Lett. 848, L12 (2017).

[5] M. Shibata, Phys. Rev. Lett. 94, 201101 (2005).

[6] L. Baiotti, B. Giacomazzo, and L. Rezzolla, Phys. Rev. D 78, 084033 (2008).

[7] K. Hotokezaka, K. Kyutoku, H. Okawa, M. Shibata, and K. Kiuchi, Phys. Rev. D 83, 124008 (2011).

[8] A. Bauswein, T. W. Baumgarte, and H.-T. Janka, Phys. Rev. Lett. 111, 131101 (2013).

[9] J. A. Faber and F. A. Rasio, Living Rev. Relativity 15, 8 (2012).

[10] L. Baiotti and L. Rezzolla, Rep. Prog. Phys. 80, 096901 (2017).

[11] V. Paschalidis and N. Stergioulas, Living Rev. Relativity 20, 7 (2017).

[12] J. L. Friedman, Int. J. Mod. Phys. D 27, 1843018 (2018).

[13] A. Bauswein and N. Stergioulas, J. Phys. G 46, 113002 (2019).

[14] L. Baiotti, Prog. Part. Nucl. Phys. 109, 103714 (2019).

[15] M. D. Duez and Y. Zlochower, Rep. Prog. Phys. 82, 016902 (2019).

[16] M. Lucca and L. Sagunski, J. High Energy Astrophys. 27, 33 (2020).

[17] D. Radice, S. Bernuzzi, and A. Perego, Annu. Rev. Nucl. Part. Sci. 70 (2020).

[18] B. P. Abbott, R. Abbott, T. D. Abbott, F. Acernese, K. Ackley, C. Adams, T. Adams, P. Addesso, R.X. Adhikari, V. B. Adya et al., Phys. Rev. X 9, 011001 (2019). 
[19] J. Clark, A. Bauswein, L. Cadonati, H.-T. Janka, C. Pankow, and N. Stergioulas, Phys. Rev. D 90, 062004 (2014).

[20] A. Torres-Rivas, K. Chatziioannou, A. Bauswein, and J. A. Clark, Phys. Rev. D 99, 044014 (2019).

[21] A. Bauswein, S. Goriely, and H.-T. Janka, Astrophys. J. 773, 78 (2013).

[22] K. Hotokezaka, K. Kiuchi, K. Kyutoku, H. Okawa, Y.-i. Sekiguchi, M. Shibata, and K. Taniguchi, Phys. Rev. D 87, 024001 (2013).

[23] B. Margalit and B. D. Metzger, Astrophys. J. Lett. 880, L15 (2019).

[24] B. D. Metzger, arXiv:1710.05931.

[25] M. Ruiz and S. L. Shapiro, Phys. Rev. D 96, 084063 (2017).

[26] M. W. Coughlin, T. Dietrich, B. Margalit, and B. D. Metzger, Mon. Not. R. Astron. Soc. 489, L91 (2019).

[27] V. Paschalidis and M. Ruiz, Phys. Rev. D 100, 043001 (2019).

[28] H.-J. Lü, J. Shen, L. Lan, J. Rice, W.-H. Lei, and E.-W. Liang, Mon. Not. R. Astron. Soc. 486, 4479 (2019).

[29] R. Gill, A. Nathanail, and L. Rezzolla, Astrophys. J. 876, 139 (2019).

[30] R. J. Foley, D. A. Coulter, C. D. Kilpatrick, A. L. Piro, E. Ramirez-Ruiz, and J. Schwab, Mon. Not. R. Astron. Soc. 494, 190 (2020).

[31] M. W. Coughlin, T. Dietrich, S. Antier, M. Bulla, F. Foucart, K. Hotokezaka, G. Raaijmakers, T. Hinderer, and S. Nissanke, Mon. Not. R. Astron. Soc. 492, 863 (2020).

[32] M. Agathos, F. Zappa, S. Bernuzzi, A. Perego, M. Breschi, and D. Radice, Phys. Rev. D 101, 044006 (2020).

[33] A. Chen, N. K. Johnson-McDaniel, T. Dietrich, and R. Dudi, Phys. Rev. D 101, 103008 (2020).

[34] C. J. Krüger and F. Foucart, Phys. Rev. D 101, 103002 (2020).

[35] S. Antier, S. Agayeva, V. Aivazyan, S. Alishov, E. Arbouch, A. Baransky, K. Barynova, J. M. Bai, S. Basa, S. Beradze et al., Mon. Not. R. Astron. Soc. 492, 3904 (2020).

[36] A. Nathanail, Astrophys. J. 892, 35 (2020).

[37] A. Bauswein, O. Just, H.-T. Janka, and N. Stergioulas, Astrophys. J. Lett. 850, L34 (2017).

[38] S. Köppel, L. Bovard, and L. Rezzolla, Astrophys. J. Lett. 872, L16 (2019).

[39] C. D. Capano, I. Tews, S. M. Brown, B. Margalit, S. De, S. Kumar, D. A. Brown, B. Krishnan, and S. Reddy, Nat. Astron. 4, 625 (2020).

[40] D. Radice, A. Perego, F. Zappa, and S. Bernuzzi, Astrophys. J. Lett. 852, L29 (2018).

[41] D. Radice and L. Dai, Eur. Phys. J. A 55, 50 (2019).

[42] A. Bauswein, N.-U.F. Bastian, D. Blaschke, K. Chatziioannou, J. A. Clark, T. Fischer, H.-T. Janka, O. Just, M. Oertel, and N. Stergioulas, AIP Conf. Proc. 2127, 020013 (2019).

[43] J. Antoniadis, P. C. C. Freire, N. Wex, T. M. Tauris, R. S. Lynch, M. H. van Kerkwijk, M. Kramer, C. Bassa, V. S. Dhillon, T. Driebe et al., Science 340, 1233232 (2013).

[44] Z. Arzoumanian, A. Brazier, S. Burke-Spolaor, S. Chamberlin, S. Chatterjee, B. Christy, J. M. Cordes,
N. J. Cornish, F. Crawford, H. T. Cromartie et al., Astrophys. J. Suppl. Ser. 235, 37 (2018).

[45] H. T. Cromartie et al., Nat. Astron. 4, 72 (2020).

[46] P. D. Lasky, B. Haskell, V. Ravi, E. J. Howell, and D. M. Coward, Phys. Rev. D 89, 047302 (2014).

[47] S. Lawrence, J. G. Tervala, P. F. Bedaque, and M. C. Miller, Astrophys. J. 808, 186 (2015).

[48] C. L. Fryer, K. Belczynski, E. Ramirez-Ruiz, S. Rosswog, G. Shen, and A. W. Steiner, Astrophys. J. 812, 24 (2015).

[49] B. Margalit and B. D. Metzger, Astrophys. J. Lett. 850, L19 (2017).

[50] M. Shibata, S. Fujibayashi, K. Hotokezaka, K. Kiuchi, K. Kyutoku, Y. Sekiguchi, and M. Tanaka, Phys. Rev. D 96, 123012 (2017).

[51] L. Rezzolla, E. R. Most, and L. R. Weih, Astrophys. J. Lett. 852, L25 (2018).

[52] M. Ruiz, S. L. Shapiro, and A. Tsokaros, Phys. Rev. D 97, 021501(R) (2018).

[53] S. Ai, H. Gao, and B. Zhang, Astrophys. J. 893, 146 (2020).

[54] M. C. Miller, C. Chirenti, and F. K. Lamb, Astrophys. J. 888, 12 (2020).

[55] D.-S. Shao, S.-P. Tang, X. Sheng, J.-L. Jiang, Y.-Z. Wang, Z.-P. Jin, Y.-Z. Fan, and D.-M. Wei, Phys. Rev. D 101, 063029 (2020).

[56] F. Özel and P. Freire, Annu. Rev. Astron. Astrophys. 54, 401 (2016).

[57] J. M. Lattimer and M. Prakash, Phys. Rep. 621, 127 (2016).

[58] M. Oertel, M. Hempel, T. Klähn, and S. Typel, Rev. Mod. Phys. 89, 015007 (2017).

[59] K. Sumiyoshi, S. Yamada, H. Suzuki, and S. Chiba, Phys. Rev. Lett. 97, 091101 (2006).

[60] T. Fischer, S. C. Whitehouse, A. Mezzacappa, F. K. Thielemann, and M. Liebendörfer, Astron. Astrophys. 499, 1 (2009).

[61] E. O'Connor and C. D. Ott, Astrophys. J. 730, 70 (2011).

[62] A. W. Steiner, M. Hempel, and T. Fischer, Astrophys. J. 774, 17 (2013).

[63] T. Fischer, M. Hempel, I. Sagert, Y. Suwa, and J. Schaffner-Bielich, Eur. Phys. J. A 50, 46 (2014).

[64] A.d. S. Schneider, E. O'Connor, E. Granqvist, A. Betranhandy, and S. M. Couch, Astrophys. J. 894, 4 (2020).

[65] R. Oechslin, K. Uryū, G. Poghosyan, and F. K. Thielemann, Mon. Not. R. Astron. Soc. 349, 1469 (2004).

[66] V. Paschalidis, K. Yagi, D. Alvarez-Castillo, D. B. Blaschke, and A. Sedrakian, Phys. Rev. D 97, 084038 (2018).

[67] E. R. Most, L. J. Papenfort, V. Dexheimer, M. Hanauske, S. Schramm, H. Stöcker, and L. Rezzolla, Phys. Rev. Lett. 122, 061101 (2019).

[68] A. N. Bauswein, N.-U.F. Bastian, D. B. Blaschke, K. Chatziioannou, J. A. Clark, T. Fischer, and M. Oertel, Phys. Rev. Lett. 122, 061102 (2019).

[69] S. Han, M. A. A. Mamun, S. Lalit, C. Constantinou, and M. Prakash, Phys. Rev. D 100, 103022 (2019).

[70] J.-E. Christian, A. Zacchi, and J. Schaffner-Bielich, Phys. Rev. D 99, 023009 (2019).

[71] M. Sieniawska, W. Turczański, M. Bejger, and J. L. Zdunik, Astron. Astrophys. 622, A174 (2019). 
[72] G. F. Burgio, A. Drago, G. Pagliara, H.-J. Schulze, and J.-B. Wei, Astrophys. J. 860, 139 (2018).

[73] A. Drago and G. Pagliara, Astrophys. J. Lett. 852, L32 (2018).

[74] V. Dexheimer, L. T. T. Soethe, J. Roark, R. O. Gomes, S. O. Kepler, and S. Schramm, Int. J. Mod. Phys. E 27, 1830008 (2018).

[75] K. Chatziioannou and S. Han, Phys. Rev. D 101, 044019 (2020).

[76] H.-Y. Chen, P. M. Chesler, and A. Loeb, Astrophys. J. Lett. 893, L4 (2020).

[77] M. G. Alford, S. Han, and K. Schwenzer, J. Phys. G 46, 114001 (2019).

[78] R. De Pietri, A. Drago, A. Feo, G. Pagliara, M. Pasquali, S. Traversi, and G. Wiktorowicz, Astrophys. J. 881, 122 (2019).

[79] L. R. Weih, M. Hanauske, and L. Rezzolla, Phys. Rev. Lett. 124, 171103 (2020).

[80] M. G. Orsaria, G. Malfatti, M. Mariani, I. F. Ranea-Sandoval, F. García, W. M. Spinella, G. A. Contrera, G. Lugones, and F. Weber, J. Phys. G 46, 073002 (2019).

[81] G. Montaña, L. Tolós, M. Hanauske, and L. Rezzolla, Phys. Rev. D 99, 103009 (2019).

[82] S. Han and A. W. Steiner, Phys. Rev. D 99, 083014 (2019).

[83] D. E. Alvarez-Castillo, D. B. Blaschke, A. G. Grunfeld, and V. P. Pagura, Phys. Rev. D 99, 063010 (2019).

[84] J. J. Li, A. Sedrakian, and M. Alford, Phys. Rev. D 101, 063022 (2020).

[85] J. P. Pereira, M. Bejger, N. Andersson, and F. Gittins, Astrophys. J. 895, 28 (2020).

[86] S. Blacker, N.-U. F. Bastian, A. Bauswein, D. B. Blaschke, T. Fischer, M. Oertel, T. Soultanis, and S. Typel, arXiv:2006.03789.

[87] A. Bazavov, T. Bhattacharya, C. DeTar, H.-T. Ding, S. Gottlieb, R. Gupta, P. Hegde, U. M. Heller, F. Karsch, E. Laermann et al., Phys. Rev. D 90, 094503 (2014).

[88] S. Borsányi, Z. Fodor, C. Hoelbling, S. D. Katz, S. Krieg, and K. K. Szabó, Phys. Lett. B 730, 99 (2014).

[89] The CBM Physics Book, edited by B. Friman, C. Höhne, J. Knoll, S. Leupold, J. Randrup, R. Rapp, and P. Senger, Lecture Notes in Physics Vol. 814 (Springer Verlag, Berlin, 2011).

[90] D. Blaschke, J. Aichelin, E. Bratkovskaya, V. Friese, M. Gazdzicki, J. Randrup, O. Rogachevsky, O. Teryaev, and V. Toneev, Eur. Phys. J. A 52, 267 (2016).

[91] J. Adamczewski-Musch et al. (HADES Collaboration), Nat. Phys. 15, 1040 (2019).

[92] B. P. Abbott, R. Abbott, T. D. Abbott, F. Acernese, K. Ackley, C. Adams, T. Adams, P. Addesso, R. X. Adhikari, V. B. Adya et al., Astrophys. J. Lett. 851, L16 (2017).

[93] R. Dudi, F. Pannarale, T. Dietrich, M. Hannam, S. Bernuzzi, F. Ohme, and B. Brügmann, Phys. Rev. D 98, 084061 (2018).

[94] H. Yang, V. Paschalidis, K. Yagi, L. Lehner, F. Pretorius, and N. Yunes, Phys. Rev. D 97, 024049 (2018).

[95] K. W. Tsang, T. Dietrich, and C. Van Den Broeck, Phys. Rev. D 100, 044047 (2019).
[96] M. Breschi, S. Bernuzzi, F. Zappa, M. Agathos, A. Perego, D. Radice, and A. Nagar, Phys. Rev. D 100, 104029 (2019).

[97] J. Isenberg and J. Nester, in General Relativity and Gravitation. Vol. 1. One Hundred Years after the Birth of Albert Einstein, edited by A. Held (Plenum Press, New York, 1980), p. 23.

[98] J. R. Wilson, G. J. Mathews, and P. Marronetti, Phys. Rev. D 54, 1317 (1996).

[99] R. Oechslin, S. Rosswog, and F.-K. Thielemann, Phys. Rev. D 65, 103005 (2002).

[100] R. Oechslin, H.-T. Janka, and A. Marek, Astron. Astrophys. 467, 395 (2007).

[101] A. Bauswein, H.-T. Janka, and R. Oechslin, Phys. Rev. D 82, 084043 (2010).

[102] A. Bauswein, H.-T. Janka, K. Hebeler, and A. Schwenk, Phys. Rev. D 86, 063001 (2012).

[103] A. Bauswein, N. Stergioulas, and H.-T. Janka, Phys. Rev. D 90, 023002 (2014).

[104] A. Bauswein et al. (in preparation).

[105] S. Banik, M. Hempel, and D. Bandyopadhyay, Astrophys. J. Suppl. Ser. 214, 22 (2014).

[106] M. Fortin, M. Oertel, and C. Providência, Pub. Astron. Soc. Aust. 35 (2018).

[107] M. Marques, M. Oertel, M. Hempel, and J. Novak, Phys. Rev. C 96, 045806 (2017).

[108] M. Hempel and J. Schaffner-Bielich, Nucl. Phys. A837, 210 (2010).

[109] S. Typel, G. Röpke, T. Klähn, D. Blaschke, and H. H. Wolter, Phys. Rev. C 81, 015803 (2010).

[110] S. Typel, Phys. Rev. C 71, 064301 (2005).

[111] D. Alvarez-Castillo, A. Ayriyan, S. Benic, D. Blaschke, H. Grigorian, and S. Typel, Eur. Phys. J. A 52, 69 (2016).

[112] A. Akmal, V.R. Pandharipande, and D. G. Ravenhall, Phys. Rev. C 58, 1804 (1998).

[113] S. Goriely, N. Chamel, and J. M. Pearson, Phys. Rev. C 82, 035804 (2010).

[114] R. B. Wiringa, V. Fiks, and A. Fabrocini, Phys. Rev. C 38, 1010 (1988).

[115] J. M. Lattimer and F. D. Swesty, Nucl. Phys. A535, 331 (1991).

[116] G. Shen, C. J. Horowitz, and S. Teige, Phys. Rev. C 83, 035802 (2011).

[117] G. A. Lalazissis, J. König, and P. Ring, Phys. Rev. C 55, 540 (1997).

[118] M. Hempel, T. Fischer, J. Schaffner-Bielich, and M. Liebendörfer, Astrophys. J. 748, 70 (2012).

[119] F. Douchin and P. Haensel, Astron. Astrophys. 380, 151 (2001).

[120] H. Müther, M. Prakash, and T. L. Ainsworth, Phys. Lett. B 199, 469 (1987).

[121] M. Alford, M. Braby, M. Paris, and S. Reddy, Astrophys. J. 629, 969 (2005).

[122] L. Engvik, E. Osnes, M. Hjorth-Jensen, G. Bao, and E. Ostgaard, Astrophys. J. 469, 794 (1996).

[123] A. S. Schneider, C. Constantinou, B. Muccioli, and M. Prakash, Phys. Rev. C 100, 025803 (2019).

[124] J. S. Read, B. D. Lackey, B. J. Owen, and J. L. Friedman, Phys. Rev. D 79, 124032 (2009).

[125] B. D. Lackey, M. Nayyar, and B. J. Owen, Phys. Rev. D 73, 024021 (2006). 
[126] N. K. Glendenning, Astrophys. J. 293, 470 (1985).

[127] Y. Sugahara and H. Toki, Nucl. Phys. A579, 557 (1994).

[128] H. Toki, D. Hirata, Y. Sugahara, K. Sumiyoshi, and I. Tanihata, Nucl. Phys. A588, c357 (1995).

[129] M. A. R. Kaltenborn, N.-U. F. Bastian, and D. B. Blaschke, Phys. Rev. D 96, 056024 (2017).

[130] N.-U. Bastian, D. Blaschke, T. Fischer, and G. Röpke, Universe 4, 67 (2018).

[131] M. Cierniak, T. Klähn, T. Fischer, and N.-U. Bastian, Universe 4, 30 (2018).

[132] T. Fischer, N.-U.F. Bastian, M.-R. Wu, P. Baklanov, E. Sorokina, S. Blinnikov, S. Typel, T. Klähn, and D. B. Blaschke, Nat. Astron. 2, 980 (2018).

[133] See Supplemental Material at http://link.aps.org/ supplemental/10.1103/PhysRevLett.125.141103 for information on simulations, EOSs, fit formulae and the influence of the mass binary mass ratio on $M_{\text {thres }}$.

[134] T. Klähn and T. Fischer, Astrophys. J. 810, 134 (2015).

[135] Y. Nambu and G. Jona-Lasinio, Phys. Rev. 122, 345 (1961).

[136] S. Klevansky, Rev. Mod. Phys. 64, 649 (1992).

[137] T. Klähn, T. Fischer, and M. Hempel, Astrophys. J. 836, 89 (2017).

[138] D. S. Balsara, J. Comput. Phys. 121, 357 (1995).

[139] J. S. Read, C. Markakis, M. Shibata, K. Uryū, J. D. E. Creighton, and J. L. Friedman, Phys. Rev. D 79, 124033 (2009).

[140] W. Del Pozzo, T. G. F. Li, M. Agathos, C. Van Den Broeck, and S. Vitale, Phys. Rev. Lett. 111, 071101 (2013).

[141] J. S. Read, L. Baiotti, J. D. E. Creighton, J. L. Friedman, B. Giacomazzo, K. Kyutoku, C. Markakis, L. Rezzolla, M. Shibata, and K. Taniguchi, Phys. Rev. D 88, 044042 (2013).

[142] L. Wade, J. D. E. Creighton, E. Ochsner, B. D. Lackey, B. F. Farr, T. B. Littenberg, and V. Raymond, Phys. Rev. D 89, 103012 (2014).

[143] M. Agathos, J. Meidam, W. Del Pozzo, T. G. F. Li, M. Tompitak, J. Veitch, S. Vitale, and C. Van Den Broeck, Phys. Rev. D 92, 023012 (2015).

[144] K. Chatziioannou, K. Yagi, A. Klein, N. Cornish, and N. Yunes, Phys. Rev. D 92, 104008 (2015).
[145] K. Hotokezaka, K. Kyutoku, Y.-i. Sekiguchi, and M. Shibata, Phys. Rev. D 93, 064082 (2016).

[146] K. Chatziioannou, C.-J. Haster, and A. Zimmerman, Phys. Rev. D 97, 104036 (2018).

[147] S. De, D. Finstad, J. M. Lattimer, D. A. Brown, E. Berger, and C. M. Biwer, Phys. Rev. Lett. 121, 091102 (2018).

[148] T. Hinderer, Astrophys. J. 677, 1216 (2008).

[149] T. Hinderer, B. D. Lackey, R. N. Lang, and J. S. Read, Phys. Rev. D 81, 123016 (2010).

[150] T. Damour and A. Nagar, Phys. Rev. D 81, 084016 (2010).

[151] M. C. Miller, F. K. Lamb, A. J. Dittmann, S. Bogdanov, Z. Arzoumanian, K. C. Gendreau, S. Guillot, A. K. Harding, W. C. G. Ho, J. M. Lattimer et al., Astrophys. J. Lett. 887, L24 (2019).

[152] T. E. Riley, A. L. Watts, S. Bogdanov, P. S. Ray, R. M. Ludlam, S. Guillot, Z. Arzoumanian, C. L. Baker, A. V. Bilous, D. Chakrabarty et al., Astrophys. J. Lett. 887, L21 (2019).

[153] G. Raaijmakers, S. K. Greif, T. E. Riley, T. Hinderer, K. Hebeler, A. Schwenk, A. L. Watts, S. Nissanke, S. Guillot, J. M. Lattimer et al., Astrophys. J. Lett. 893, L21 (2020).

[154] A. Bauswein and N. Stergioulas, Mon. Not. R. Astron. Soc. 471, 4956 (2017).

[155] F. Zappa, S. Bernuzzi, D. Radice, A. Perego, and T. Dietrich, Phys. Rev. Lett. 120, 111101 (2018).

[156] S. Bernuzzi, M. Breschi, B. Daszuta, A. Endrizzi, D. Logoteta, V. Nedora, A. Perego, D. Radice, F. Schianchi, F. Zappa et al., Mon. Not. R. Astron. Soc. 497, 1488 (2020).

[157] K. Kiuchi, K. Kyutoku, M. Shibata, and K. Taniguchi, Astrophys. J. Lett. 876, L31 (2019).

[158] P. Danielewicz, R. Lacey, and W. G. Lynch, Science 298, 1592 (2002).

[159] C. Y. Tsang, M. B. Tsang, P. Danielewicz, W. G. Lynch, and F. J. Fattoyev, arXiv:1807.06571.

[160] J. M. Lattimer and Y. Lim, Astrophys. J. 771, 51 (2013).

[161] T. Krüger, I. Tews, K. Hebeler, and A. Schwenk, Phys. Rev. C 88, 025802 (2013).

[162] B. P. Abbott, R. Abbott, T. D. Abbott, F. Acernese, K. Ackley, C. Adams, T. Adams, P. Addesso, R. X. Adhikari, V. B. Adya et al., Phys. Rev. Lett. 121, 161101 (2018). 